\title{
PERPETUAL OPTIONS AND CANADIZATION THROUGH FLUCTUATION THEORY
}

\author{
By A. E. KYPRIANOU AND M. R. Pistorius \\ Utrecht University
}

\begin{abstract}
In this article it is shown that one is able to evaluate the price of perpetual calls, puts, Russian and integral options directly as the Laplace transform of a stopping time of an appropriate diffusion using standard fluctuation theory. This approach is offered in contrast to the approach of optimal stopping through free boundary problems. Following ideas of Carr [Rev. Fin. Studies 11 (1998) 597-626], we discuss the Canadization of these options as a method of approximation to their finite time counterparts. Fluctuation theory is again used in this case.
\end{abstract}

1. Introduction. We begin by introducing the standard stochastic model of a complete arbitrage free market. The market consists of a bond and a risky asset. The value of the bond $B=\left\{B_{t}: t \geq 0\right\}$ evolves in time deterministically such that

$$
B_{t}=B_{0} e^{r t}, \quad B_{0}>0, r \geq 0, t \geq 0 .
$$

The value of the risky asset $S=\left\{S_{t}: t \geq 0\right\}$ is defined on a filtered probability space $(\Omega, \mathcal{F}, \mathbf{F}, \mathbb{P})$ with the following components. $\Omega$ is the space of continuous functions $\omega=\left\{\omega_{t}\right\}_{t \geq 0}$, from $[0, \infty)$ to $\mathbb{R}$ with $\omega_{0}=0$. $\mathcal{F}$ is the smallest $\sigma$-algebra on $\Omega$ such that for every $t \geq 0$, the map $\omega \mapsto \omega_{t}$ of $\Omega$ to $\mathbb{R}$ is $\mathcal{F} / \mathcal{B}$-measurable, where $\mathscr{B}$ is the Borel- $\sigma$-algebra on $\mathbb{R}$. The probability measure $\mathbb{P}$ on $(\Omega, \mathcal{F})$ is such that $W=W(\omega)=\left\{\omega_{t}: t \geq 0\right\}=\left\{W_{t}: t \geq 0\right\}$ is a Wiener process starting from the origin. Let $\mathcal{F}_{t}^{0}$ be the $\sigma$-algebra generated by $W$ up to time $t$, then the filtration $\mathbf{F}$ is a flow of $\sigma$-algebras $\left\{\mathscr{F}_{t}: t \geq 0\right\}$, which are equal to the closure of $\bigcap_{s>t} \mathcal{F}_{s}^{0}$ by the $\mathbb{P}$-null sets of $\mathcal{F}$. The dynamics of the risky asset under $\mathbb{P}$ are given by an exponential of a Brownian motion with drift

$$
S_{t}=s \exp \left\{\sigma W_{t}+\mu t\right\},
$$

where $s>0, \sigma>0$ and $\mu \in \mathbb{R}$.

An option is a contract between the seller and the buyer, in which the buyer receives payments of the seller if certain events happen. Options may be divided into two classes: American-type options, which can be exercised at any time before the expiration date and European-type options, which have exercise only at expiration. A perpetual option is an American-type option with no expiration date. The buyer of a perpetual has the right to exercise it at any time $t$ and receive

Received November 2000; revised November 2002.

AMS 2000 subject classifications. Primary 60G40, 60G99; secondary 60J65.

Key words and phrases. Option pricing, perpetual option, call option, put option, Russian option, integral option, stopping time, Laplace transform, Brownian motion, Bessel process. 
then a payment $\pi_{t}$, which depends in some way on the underlying stock price $S$. Note that the zero time point is always taken to be the instant at which the contract commences. Examples of perpetual options are the call, the put, the Russian option $[19,20]$, and the integral option [11], with payments $\pi^{c}, \pi^{p}, \pi^{r}, \pi^{i}$, respectively,

$$
\begin{aligned}
\pi_{t}^{p} & =e^{-\lambda t}\left(K-S_{t}\right)^{+}, & \pi_{t}^{c} & =e^{-\lambda t}\left(S_{t}-K\right)^{+}, \\
\pi_{t}^{r} & =e^{-\lambda t} \max \left\{\max _{u \leq t} S_{u}, s \psi\right\}, & \pi_{t}^{i} & =e^{-\lambda t}\left[\int_{0}^{t} S_{u} d u+s \varphi\right],
\end{aligned}
$$

where $\lambda, K, \psi, \varphi>0$ are constants.

REMARK. The parameter $K$ is called the strike price, $s$ is usually taken as the value of the stock at time zero and we use $y^{+}$to denote $\max \{y, 0\}$. The parameter $\lambda$ can be considered as a continuous dividend rate. In order for the arbitrage-free price of the Russian, call and integral perpetual option to be finite, $\lambda$ has to be positive, whereas the price of the put remains finite for $\lambda=0$. See also $[7,22,20]$. Note that $s \psi$ can be understood to be the supremum of the risky asset price process over some precontract period. Likewise, $s \varphi$ can be understood to be the integral of the stock price over some precontract period.

The payoffs of the perpetual call and put differ fundamentally from that of the Russian and integral option. The payoffs of call and put only depend on the value of the underlying stock $S$ at the exercise time, whereas the Russian and integral options are path dependent options. That is to say that the payoff $\pi_{t}$ depends on the whole path of the stock price $S$ from some instant at or before the contract begins and up to time $t$.

Two fundamental questions that can be asked of American-type perpetual options are:

Q1. What is the arbitrage-free price of the option?

$\mathrm{Q} 2$. What is an optimal time to exercise?

Theorems 1.1 and 1.2 (see also, e.g., [22] and [10]) give answers to these questions, but in a form that is not handy from an applied perspective. In order to state these theorems, we must first introduce a little more notation.

Throughout this article we shall use the letters $s$ and $x$ with the assumed relation

$$
s=\exp \{\sigma x\}
$$

to represent the relationship between the starting points of $S$ and $W$. We introduce the measure $\mathbb{P}_{x}$ which is a translation of the measure $\mathbb{P}$ such that under $\mathbb{P}_{x}, W$ is a Wiener process with inital position $W_{0}=x$. Now introduce the measure $\mathbb{P}_{x}^{\gamma}$ under which $W_{t}-\gamma t$ is a Wiener process starting from $x$. The measures $\mathbb{P}_{x}^{\gamma}$ and $\mathbb{P}_{x}$ are related through the Girsanov change of measure

$$
\left.\frac{d \mathbb{P}_{x}^{\gamma}}{d \mathbb{P}_{x}}\right|_{\mathcal{F}_{t}}=\exp \left\{\gamma\left(W_{t}-x\right)-\frac{1}{2} \gamma^{2} t\right\} \text {. }
$$


Henceforth it is understood that $\mathbb{E}_{x}^{\gamma}$ refers to expectation with respect to $\mathbb{P}_{x}^{\gamma}$. Note the value of the risky asset under $\mathbb{P}^{\mu / \sigma}$ satisfies $S_{t}=\exp \left\{\sigma W_{t}\right\}$.

Finally let $\mathcal{T}_{t, \infty}$ be the set of $\mathbf{F}$-stopping times valued in $[t, \infty)$ and $\overline{\mathcal{T}}_{t, \infty}$ the set of $\mathbf{F}$-stopping times valued in $[t, \infty]$ where $t \geq 0$.

Suppose now that $\pi=\left\{\pi_{t}: t \geq 0\right\}$ is an $\mathbf{F}$-adapted sequence of nonnegative payments. The following well-established theorem addresses Q1 when the option holder has even the right never to exercise, corresponding to the case that their exercise time is infinite with possibly positive probability.

THEOREM 1.1. The artbitrage-free price $\Pi(t, s)$ for an American-type perpetual option at time $t$ into the contract, with payments $\pi$ and $S$ starting at $s$ satisfies

$$
\Pi(t, s)=\underset{\tau \in \overline{\mathcal{T}}_{t, \infty}}{\operatorname{ess} \sup } \mathbb{E}_{x}^{(r / \sigma-\sigma / 2)}\left[e^{-r(\tau-t)} \pi_{\tau} \mid \mathcal{F}_{t}\right]
$$

In particular, the arbitrage-free price of the option is given by

$$
\sup _{\tau \in \overline{\mathcal{T}}_{0, \infty}} \mathbb{E}_{x}^{(r / \sigma-\sigma / 2)}\left[e^{-r \tau} \pi_{\tau}\right] .
$$

If we formulate the problem insisting that the buyer must exercise within an almost surely finite time, then exactly the same result holds except that $\overline{\mathcal{T}}_{t, \infty}$ should be replaced by $\mathcal{T}_{t, \infty}$.

The next theorem, taken from [22], addresses Q2.

THEOREM 1.2. Suppose that the payments $\pi$ are $\mathcal{F}_{t}$-measurable, cadlag, without negative jumps and

$$
\left\{e^{-r \tau} \pi_{\tau}: \tau \in \overline{\mathcal{T}}_{0, \infty}\right\}
$$

is uniformly integrable with respect to $\mathbb{P}^{(r / \sigma-\sigma / 2)}$. Then

$$
\tau^{*}=\inf \left\{t \geq 0: \Pi(t, s) \leq \pi_{t}\right\}
$$

is an optimal exercise time for (1.4).

Again, when the problem of pricing is reformulated so that the buyer must exercise within an almost surely finite time, in the above theorem we can replace $\overline{\mathcal{T}}_{0, \infty}$ by $\mathcal{T}_{0, \infty}$.

In reviewing the literature concerning perpetual options, one finds two dominant methods that are used for their evaluation. 
Free boundary problem approach. The first method has been nicely characterized in a series of papers $[22,20,11]$ that appeared all together in Volume 39 of Theory of Probability and Its Applications. However, its origin can be traced back as far as McKean's paper [15] in 1965. In these papers an approach based on free boundary problems, sometimes called Stefan problems, is applied to perpetual American call and put options, Russian options and integral options. Based on heuristic reasoning, the solution to an appropriate free boundary problem is taken as a candidate price for the option at hand. Then this solution is shown to be equal to the supremum (1.4) as a consequence of it being a solution to the free boundary problem.

Fluctuation theory appoach. The second approach [22, 12], used for evaluating American call and put options, consists of proving that the optimal stopping time has the form of a hitting time of the stock price at some level, say $a$. Given that $\left(K-S_{t}\right)^{+}$[or indeed $\left(S_{t}-K\right)^{+}$] is constant at such a hitting time, the price of the option is essentially proportional to the Laplace transform of the hitting time optimized over the level $a$. The computations for this procedure are very elementary once the optimal stopping time is realized as a hitting time.

In the case of the Russian perpetual option, it is also worth mentioning the paper [9]. In this paper the authors use two important properties to recover the price of the Russian perpetual. The first is that for a continuous Markov processes $Z$, if $\tau_{v}$ is a hitting time of $Z$ then the expectation $E_{z}\left(e^{-\lambda \tau_{v}} Z_{\tau_{v}}\right)$ is a solution to a certain elliptic equation with boundary conditions. The second fact is the strong Markov property. These two essentially are enough to show that the optimal stopping time is that of a hitting time of an appropriate diffusion and also give the analytical form of the solution.

Below we give the conclusion of both the fluctuation theory and free boundary methods for perpetual calls and puts and the conclusion achieved by the first of these two methods for perpetual Russian and integral options. Recall that $r$ and $\sigma$ are parameters of the market $(B, S)$ and $\lambda$ is a parameter appearing in the claims outlined in (1.2) and (1.3).

Let $x_{1}<0<x_{2}$ be the two roots of the quadratic equation

$$
x^{2}-\left(1-\frac{2 r}{\sigma^{2}}\right) x-\left(\frac{2 \lambda+2 r}{\sigma^{2}}\right)=0 .
$$

THEOREM 1.3. The arbitrage-free price of a perpetual call and put at time $t$ into the contract, $\Pi^{\text {call }}(t, s)$ and $\Pi^{\text {put }}(t, s)$, with payoff $\pi^{\mathrm{c}}$ and $\pi^{\mathrm{p}}$, respectively, are given by

$$
\Pi^{\text {call }}(t, s)=e^{-\lambda t} \Pi^{\mathrm{C}}\left(S_{t}\right) \quad \text { and } \quad \Pi^{\mathrm{put}}(t, s)=e^{-\lambda t} \Pi^{\mathrm{P}}\left(S_{t}\right),
$$

where

$$
\Pi^{\mathrm{C}}(s)= \begin{cases}\left(s_{2}-K\right)\left(s / s_{2}\right)^{x_{2}}, & \text { if } s<s_{2}, \\ s-K, & \text { if } s \geq s_{2},\end{cases}
$$


and

$$
\Pi^{\mathrm{P}}(s)= \begin{cases}\left(K-s_{1}\right)\left(s / s_{1}\right)^{x_{1}}, & \text { if } s>s_{1}, \\ K-s, & \text { if } s \leq s_{1} .\end{cases}
$$

Here

$$
s_{1}=K \frac{x_{1}}{x_{1}-1}<K \frac{x_{2}}{x_{2}-1}=s_{2}
$$

are the optimal exercise boundaries. That is to say that the holder should exercise if the value of the asset exceeds or falls below $s_{2}$ and $s_{1}$ in the case of the call and put, respectively.

Consider now the equation

$$
y^{2}-\left(1+\frac{2 r}{\sigma^{2}}\right) y-\left(\frac{2 \lambda}{\sigma^{2}}\right)=0
$$

with roots $y_{1}<0<1<y_{2}$.

THEOREM 1.4. The arbitrage-free price $\Pi^{\mathrm{russ}}(t, s, \psi)$ of a perpetual Russian option at time $t$ into the contract with payoff $\pi^{\mathrm{r}}$ satisfies

$$
\Pi^{\mathrm{russ}}(t, s, \psi)=e^{-\lambda t} S_{t} \Pi^{\mathrm{R}}\left(\Psi_{t}\right),
$$

where $\Psi_{t}:=\left(\sup _{0 \leq u \leq t} S_{u} \vee s \psi\right) / S_{t}$ and

$$
\Pi^{\mathrm{R}}(\psi)= \begin{cases}\tilde{\psi} \frac{y_{2} \psi^{y_{1}}-y_{1} \psi^{y_{2}}}{y_{2} \tilde{\psi}^{y_{1}}-y_{1} \tilde{\psi}^{y_{2}}}, & 1 \leq \psi<\tilde{\psi}, \\ \psi, & \psi \geq \tilde{\psi} .\end{cases}
$$

Here

$$
\tilde{\psi}=\left|\frac{y_{2}}{y_{1}} \frac{y_{1}-1}{y_{2}-1}\right|^{1 /\left(y_{2}-y_{1}\right)}
$$

is the optimal exercise boundary. That is to say that the holder should exercise if the process $\Psi_{t}$ exceeds or equals $\tilde{\psi}$.

THEOREM 1.5. The arbitrage-free price $\Pi^{\mathrm{int}}(t, s, \varphi)$ of a perpetual integral option at time $t$ into the contract with payoff $\pi^{\mathrm{i}}$ satisfies

$$
\Pi^{\mathrm{int}}(t, s, \varphi)=e^{-\lambda t} S_{t} \Pi^{\mathrm{I}}\left(\Phi_{t}\right),
$$

where $\Phi_{t}:=\left(\int_{0}^{t} S_{u} d u+\varphi s\right) / S_{t}$ and

$$
\Pi^{\mathrm{I}}(\varphi)= \begin{cases}\varphi^{*} \frac{u(\varphi)}{u\left(\varphi^{*}\right)}, & 0 \leq \varphi<\varphi^{*}, \\ \varphi, & \varphi \geq \varphi^{*},\end{cases}
$$


where

$$
u(\varphi)=\int_{0}^{\infty} e^{-2 z / \sigma^{2}} z^{-y_{2}}(1+\varphi z)^{y_{1}} d z
$$

and $\varphi^{*}$ is the root of the equation $\varphi u^{\prime}(\varphi)=u(\varphi)$. Here $\varphi^{*}$ is the optimal exercise boundary, such that the holder should exercise once the process $\Phi_{t}$ exceeds or equals $\varphi^{*}$.

In this paper we shall show that the pricing of Russian and integral perpetual options can also be reduced to evaluating a Laplace transform of the hitting time of an appropriate diffusion, followed by a simple optimization over the hitting level. These new proofs will rely heavily on fluctuation theory of Brownian motion and Bessel processes thus remaining loyal to ideas used in pricing perpetual calls and puts as explained in the second method above.

Several different proofs for pricing perpetual Russian options and one proof for the pricing of integral options already exist: $[19,20,7,11,9]$. One might therefore question the motivation behind providing alternative proofs. The first reason is that the methods used in this paper can be and have been applied in markets where the underlying is assumed to be driven by a spectrally one-sided Lévy process. The interested reader is refered to [1]. The free boundary problem approach in principle may also be applicable in this case. However, knowledge of solutions to integrodifferential equations is needed as opposed to fluctuation theory of Lévy processes. The existence of solutions to such integro-differential equations in general is less understood than the availible tools for fluctuation theory. Secondly, the fluctuation techniques also give us an approach to deal with the issue of Canadization.

The rest of this paper is organized as follows. In the next section, for the sake of completeness and later reflection, we review the derivation of the arbitrage-free price of perpetual calls and puts in the context of fluctuation theory. Continuing in this vein, in Section 3 we show how the value of the Russian perpetual option can be established in a similar way. The strength of Section 3 centers around Theorem 3.2 which evaluates the Laplace transform of a hitting time of a Brownian motion reflected at its supremum. Section 4 deals with the integral option. In this case the optimal stopping time turns out to be that of a Bessel squared process with drift. This follows from the close relationship between exponential Brownian motion and Bessel squared processes (cf. [25, 26]). This connection also appears in the study of Asian options in [8].

Recently it has been proposed by Carr in [6] that finite expiry American-type options can be approximated by a randomization of the expiry date using an independent exponential distribution. This is what Carr refers to as Canadization. The effect of randomization is to make the optimal exercise boundary a constant, just as in the perpetual case. A better approximation to a fixed time expiry than this can be made by randomizing using a sum of $n$ independent exponential distributions (hence an Erlang distribution) whose total mean is the length of the 
contract. As $n$ tends to infinity, it is possible to show convergence to the price of the finite expiry American option. These ideas work equally well for the Russian and integral option and we discuss them in Section 5.

On a final note we should say that the use of fluctuation theory, as indicated in the title of this paper, in effect constitutes only half of the pricing procedure. There is still a strength of optimal stopping theory found in Theorems 1.1 and 1.2 which give the foundation on which we build. For standard references in the context of these the reader is referred to [21], [17] and [12].

2. Perpetual call and put options. Combining Theorem 1.1 with the actual form of the system of payments for call and put (1.2), we find by a simple Markovian decompostion of the process $S_{t}$ that the price $\Pi^{\text {call }}, \Pi^{\text {put }}$ of a perpetual call and put satisfy (1.6), where

$$
\begin{aligned}
& \Pi^{\text {call }}(t, s)=e^{-\lambda t} \Pi^{\mathrm{C}}\left(S_{t}\right)=e^{-\lambda t} \sup _{\tau \in \overline{\mathcal{T}}_{0, \infty}} \mathbb{E}_{x}^{(r / \sigma-\sigma / 2)}\left[e^{-(r+\lambda) \tau}\left(S_{\tau}-K\right)^{+}\right], \\
& \Pi^{\mathrm{put}}(t, s)=e^{-\lambda t} \Pi^{\mathrm{P}}\left(S_{t}\right)=e^{-\lambda t} \sup _{\tau \in \overline{\mathcal{T}}_{0, \infty}} \mathbb{E}_{x}^{(r / \sigma-\sigma / 2)}\left[e^{-(r+\lambda) \tau}\left(K-S_{\tau}\right)^{+}\right] .
\end{aligned}
$$

PROPOSITION 2.1. The optimal stopping times in (2.1) and (2.2) are of the form

$$
\inf \left\{t \geq 0: S_{t} \geq e^{\sigma h}\right\} \quad \text { and } \quad \inf \left\{t \geq 0: S_{t} \leq e^{\sigma l}\right\}
$$

respectively, where $h$ and $l$ are real constants.

Proof. By choosing $\tau=0$, we see that $\Pi^{\mathrm{C}}(s) \geq(s-K)^{+}, \Pi^{\mathrm{P}}(s) \geq$ $(K-s)^{+}$; that is, perpetual calls and puts are always at least as valuable as the direct payoff. Noting that the function $x \mapsto(x-K)^{+}$is increasing and convex, we see $\Pi^{\mathrm{C}}(\cdot)$ is increasing and convex, since integration and taking the supremum preserve monotonicity and convexity. Furthermore, $\Pi^{\mathrm{C}}$ is bounded above by $\sup _{\tau} \mathbb{E}_{x}^{(r / \sigma-\sigma / 2)}\left[e^{-(r+\lambda) \tau} S_{\tau}\right] \leq \infty$. Similarly, by the properties of $x \mapsto(K-x)^{+}$, $\Pi^{\mathrm{P}}(\cdot)$ is bounded by $K$, decreasing and convex. Theorem 1.2 implies the optimal stopping times for the call and put are given by $\inf \left\{t \geq 0: \Pi^{\mathrm{C}}\left(S_{t}\right)=\left(S_{t}-K\right)^{+}\right\}$ and $\inf \left\{t \geq 0: \Pi^{\mathrm{P}}\left(S_{t}\right)=\left(K-S_{t}\right)^{+}\right\}$, respectively, which combined with the above remarks completes the proof.

REMARK. If we define for any Borel set $B$,

$$
\tau_{B}^{W}=\inf \left\{t \geq 0: W_{t} \in B\right\}
$$

then both the stopping times in the above proposition can be expressed, respectively, as $\tau_{[h, \infty)}^{W}$ and $\tau_{(-\infty, l]}^{W}$ under $\mathbb{P}_{x}^{(r / \sigma-\sigma / 2)}$. 
By Proposition 2.1, the supremum over all stopping times in $\overline{\mathcal{T}}_{0, \infty}$ in (2.1) and (2.2) is equal to the supremum over all hitting times $\left\{\tau_{[h, \infty)}^{W}: h \in \mathbb{R}\right\}$ and $\left\{\tau_{(-\infty, l]}^{W}: l \in \mathbb{R}\right\}$, respectively. Thanks to the continuity of Brownian motion, there is no overshoot at these stopping times. Thus the prices $\Pi^{\text {call }}, \Pi^{\text {put }}$ are given by $\Pi^{\mathrm{C}}(s)=\sup _{h \in \mathbb{R}} V_{h}^{(1)}(s)$ and $\Pi^{\mathrm{P}}(s)=\sup _{l \in \mathbb{R}} V_{l}^{(2)}(s)$ where

$$
V_{h}^{(1)}(s)= \begin{cases}\mathbb{E}_{x}^{(r / \sigma-\sigma / 2)}\left[e^{-(r+\lambda) \tau_{[h, \infty)}^{W}}\right]\left(e^{\sigma h}-K\right)^{+}, & \log s<\sigma h, \\ (s-K)^{+}, & \log s \geq \sigma h\end{cases}
$$

and

$$
V_{l}^{(2)}(s)= \begin{cases}\mathbb{E}_{x}^{(r / \sigma-\sigma / 2)}\left[e^{-(r+\lambda) \tau_{(-\infty, l]}^{W}}\right]\left(K-e^{\sigma l}\right)^{+}, & \log s>\sigma l \\ (K-s)^{+}, & \log s \leq \sigma l\end{cases}
$$

REMARK. The functions $V_{h}^{(1)}$ and $V_{l}^{(2)}$ in (2.3) and (2.4) have a clear financial interpretation. $V_{h}^{(1)}$ is the value of an option that "knocks in" on exceedance of the level $\exp \sigma h$ with call rebate; that is, the option expires as soon as the stock exceeds the level $\exp \sigma h$ and pays out then the amount $(\exp \sigma h-K)^{+}$. By optimizing over all possible values of $h$ we find the value of the perpetual call. Similarly, $V_{l}^{(2)}$ is the value function of an option which expires if the stock value falls below the level $\exp \sigma l$ and then pays out the amount $(K-\exp \sigma l)^{+}$.

Thus, the computation of the prices $\Pi^{\text {call }}, \Pi^{\text {put }}$ boils down to the computation of the Laplace transform of a hitting time of Brownian motion at a certain (constant) level, followed by an optimization over that level. This Laplace transform has a well-known explicit formula to be found in any standard text on Brownian motion and can, for example, easily be derived using the Wald martingale. We thus quote without reference that

$$
\mathbb{E}_{x}^{(r / \sigma-\sigma / 2)}\left[e^{-(r+\lambda) \tau_{[h, \infty)}^{W}}\right]=e^{-\sigma x_{2}(h-x)}
$$

and

$$
\mathbb{E}_{x}^{(r / \sigma-\sigma / 2)}\left[e^{-(r+\lambda) \tau_{(-\infty, l]}^{W}}\right]=e^{\sigma x_{1}(x-l)}
$$

when $h>x$ and $l<x$, respectively. Recall that $x_{1}$ and $x_{2}$ are the roots of the quadratic equation (1.5).

The proof of Theorem 1.3 follows as a simple optimization procedure in (2.3) and (2.4).

REMARK. Notice the optimal stopping times for the optimal stopping problem are not necessarily finite, depending on the sign of $r-\sigma^{2} / 2$. If, for example, $r<\sigma^{2} / 2$ and the risky asset starts below the optimal exercise value $s_{2}$, the optimal stopping time for a call is infinite with positive $\mathbb{P}^{(r / \sigma-\sigma / 2)}$-probability. Had we insisted that the holder should exercise in an almost surely finite time, there would have been no optimal exercise strategy in this case. 
3. Perpetual Russian option. Following the lead of [22], the first step in solving this problem consists in recognizing that under $\mathbb{P}_{x}^{(r / \sigma-\sigma / 2)}, s^{-1} e^{-r t} S_{t}$ acts as a Girsanov change of measure, which adds an extra drift $\sigma$ to the Wiener process $W$. If we insist now that the claimants of the Russian option must exercise within an almost surely finite time we can use the above change of measure together with Theorem 1.1 to get

$$
\Pi^{\text {russ }}(t, s, \psi)=S_{t} \underset{\tau \in \mathcal{T}_{t, \infty}}{\operatorname{ess} \sup } \mathbb{E}_{x}^{(r / \sigma+\sigma / 2)}\left[e^{-\lambda \tau} \frac{\bar{S}_{\tau} \vee \psi s}{S_{\tau}} \mid \mathcal{F}_{t}\right],
$$

where $\bar{S}_{t}:=\max _{0 \leq u \leq t} S_{u}$. Introduce the new stochastic process $\Psi=\left\{\Psi_{t}, t \geq 0\right\}$ with $\Psi_{t}=\left(\bar{S}_{t} \vee \psi s\right) / S_{t}$. Note that it can be easily verified that $\Psi$ is a Markov process (see [20]). Suppose now that the underlying Brownian motion has been running not since time zero, but since some time $-M<0$ and further that, given $\widetilde{F}_{0}$, the exponential of the current distance of the Brownian motion from its previous maximum is $\psi$. In this instance $\Psi$ can be understood to be the exponential of the excursions of a Brownian motion with drift away from its maximum given that at time zero its value is $\psi$. With this in mind, for each $\gamma \in \mathbb{R}$ let us introduce a new measure $\overline{\mathbb{P}}_{\psi}^{\gamma}$ under which we assume that $\Psi_{0}=\psi$ and that $W_{t}-\gamma t$ is a Wiener process. We shall reserve the special notation $\overline{\mathbb{P}}^{\gamma}=\overline{\mathbb{P}}_{1}^{\gamma}$. In light of the fact that $\Psi$ is a Markov process we can thus rewrite (3.1) as

$$
\Pi^{\mathrm{russ}}(t, s, \psi)=e^{-\lambda t} S_{t} \Pi^{\mathrm{R}}\left(\Psi_{t}\right)
$$

with

$$
\Pi^{\mathrm{R}}(\psi)=\sup _{\tau \in \mathcal{T}_{0, \infty}} \overline{\mathbb{E}}_{\psi}^{(r / \sigma+\sigma / 2)}\left[e^{-\lambda \tau} \Psi_{\tau}\right]
$$

where $\overline{\mathbb{E}}_{\psi}^{\gamma}$ is expectation with respect to $\overline{\mathbb{P}}_{\psi}^{\gamma}$ and, in effect, we may now take $\Psi_{t}:=\bar{S}_{t} / S_{t}$ (which is not a function of $s$ ). Moreover, on account of Theorem 1.2, the optimal stopping time in (3.2) is given by

$$
\inf \left\{s \geq 0: \Pi^{\mathrm{R}}\left(\Psi_{s}\right) \leq \Psi_{s}\right\}
$$

PROPOSITION 3.1. The optimal stopping time in (3.2) is given by

$$
\tau^{*}=\inf \left\{t \geq 0: \Psi_{s} \geq \tilde{\psi}\right\}
$$

for some constant $\tilde{\psi} \geq 0$.

PROOF. By choosing the stopping time $\tau=0$ we see that $\Pi^{\mathrm{R}}(\psi) \geq \psi$. Now note that we can write

$$
\Pi^{\mathrm{R}}(\psi)=\sup _{\tau \in \mathcal{T}_{0, \infty}} \overline{\mathbb{E}}_{1}^{(r / \sigma+\sigma / 2)}\left[e^{-\lambda \tau}\left(\bar{S}_{\tau} \vee \psi s\right) / S_{\tau}\right]
$$

where the dependency on $s$ is superficial as it disappears through cancelation in 
the ratio. Since for every $\omega \in \Omega$ the function $\psi \mapsto\left(\bar{S}_{\tau} \vee \psi s\right) / S_{\tau}$ is a convex increasing function, $\Pi^{\mathrm{R}}(\cdot)$ inherits these properties, as integration over $\omega$ and taking the supremum over $\tau$ preserve monotonicity and convexity. Combining these facts with Theorem 1.2 completes the proof of optimality of $\tau^{*}$. Finally, from the expression for the Laplace transform of a hitting time of $\Psi$ of the form (3.4), stated in the forthcoming Theorem 3.2, we deduce that the optimal level $\tilde{\psi}$ is finite [since under $\overline{\mathbb{P}}^{(r / \sigma+\sigma / 2)}$ the Laplace transform is $o\left(\tilde{\psi}^{-1}\right)(\tilde{\psi} \rightarrow \infty)$ ] and also that the stopping time $\tau^{*}$ is almost surely finite. Thus $\tau^{*} \in \mathcal{T}_{0, \infty}$.

It can now be seen that, just as in the previous section, the valuation of the Russian option can be achieved by the evaluation of the Laplace transform of a crossing time. The following theorem tells us what we need to know.

THEOREM 3.2. For Borel sets B let

$$
\tau_{B}^{\Psi}=\inf \left\{t \geq 0: \log \Psi_{t} \in B\right\} .
$$

Setting $\eta=\sqrt{2 \lambda+\gamma^{2}} / \sigma$, we have for $\log \psi \in[0, b]$ and $\gamma \in \mathbb{R}$,

$$
\overline{\mathbb{E}}_{\psi}^{\gamma}\left[e^{\left.-\lambda \tau_{[b, \infty)}^{\Psi}\right]}=\left(\frac{\psi}{e^{b}}\right)^{\gamma / \sigma} \frac{\sigma \eta \cosh (\eta \log \psi)-\gamma \sinh (\eta \log \psi)}{\sigma \eta \cosh (\eta b)-\gamma \sinh (\eta b)} .\right.
$$

Proof of TheOrem 1.4. From (3.2), Proposition 3.1, the continuity of Brownian motion and then Theorem 3.2, it follows as a matter of checking that $\Pi^{\mathrm{R}}(\psi)$ is equal to the supremum over all $m \geq 1$ of $V_{m}^{(3)}(\psi)$ where

$$
V_{m}^{(3)}(\psi)= \begin{cases}m \frac{y_{2} \psi^{y_{1}}-y_{1} \psi^{y_{2}}}{y_{2} m^{y_{1}}-y_{1} m^{y_{2}}}, & 1 \leq \psi \leq m, \\ \psi, & \psi>m .\end{cases}
$$

Here $y_{1}$ and $y_{2}$ are the two solutions to the quadratic equation (1.7). By elementary optimization we find that $\Pi^{\mathrm{R}}$ is given by (1.8).

We conclude this section by proving Theorem 3.2.

Proof of TheOREM 3.2. First we prove the identity for $\sigma=1$ and $\psi=1$. The process $\left\{\log \Psi_{t}=\bar{W}_{t}-W_{t}: t \geq 0\right\}$ can be written as the excursion process of $W$ away from its supremum $\bar{W}_{t}=\sup _{s \leq t} W_{t}$. Now let $L=\left\{L_{t}: t \geq 0\right\}$ be local time at zero of $\bar{W}-W$. It is well known that this process can be taken as simply the supremum; that is, $L=\bar{W}$. Setting $\eta=\sqrt{2 \lambda+\gamma^{2}}$, we use Girsanov's theorem to find that

$$
\begin{aligned}
\overline{\mathbb{E}}^{\gamma}\left[e^{\left.-\lambda \tau_{[b, \infty)}^{\Psi}\right]}\right. & =\overline{\mathbb{E}}\left[e^{-\left(\lambda+\gamma^{2} / 2\right) \tau_{[b, \infty)}^{\Psi}+\gamma W\left(\tau_{[b, \infty)}^{\Psi}\right)}\right]=\overline{\mathbb{E}}^{\eta}\left[e^{(\gamma-\eta) W\left(\tau_{[b, \infty)}^{\Psi}\right)}\right] \\
& =\overline{\mathbb{E}}^{\eta}\left[e^{(\gamma-\eta)\left(W\left(\tau_{[b, \infty)}^{\Psi}\right)-\bar{W}\left(\tau_{[b, \infty)}^{\Psi}\right)+\bar{W}\left(\tau_{[b, \infty)}^{\Psi}\right)\right.}\right] \\
& =e^{(\eta-\gamma) b} \overline{\mathbb{E}}^{\eta}\left[e^{(\gamma-\eta) L\left(\tau_{[b, \infty)}^{\Psi}\right)}\right]
\end{aligned}
$$


where we used that $L=\bar{W}$.

Now recall that, since $\log \Psi$ is recurrent under $\overline{\mathbb{P}}^{\eta}$, Itô theory of excursions tells us that under $\overline{\mathbb{P}}^{\eta}$ the suprema of excursions of $\log \Psi$ away from zero $\left\{h_{t}: t \geq 0\right\}$ form a Poisson point process indexed by the local time $L$. Since $L\left(\tau_{[b, \infty)}^{\Psi}\right)$ is the time in this Poisson point process at which the first excursion with height greater or equal to $b$ occurs, $L\left(\tau_{[b, \infty)}^{\Psi}\right)$ is exponentially distributed with parameter $\nu[b, \infty)$ where $v$ is the characteristic measure of the Poisson point process $h$.

In order to proceed with the right-hand side of (3.5) we need to supply an expression for $v[b, \infty)$. Under $\mathbb{P}^{\eta}$ and for $x>0$, the set $\left\{\tau_{[y, \infty)}^{W}<\tau_{(-\infty,-x]}^{W}\right\}$ coincides with the set $\left\{h_{t} \leq t+x ; 0 \leq t \leq y\right\}$ of excursions of $W$ away from its supremum, upto local time $y$, which have height smaller than $x+t$ at local time $t$. Let $N_{t}(b)$ denote the number of excursions of maximal height greater or equal to $b$ up to local time $t$. Then,

$$
\begin{aligned}
\mathbb{P}^{\eta}\left(h_{t}\right. & \leq t+x ; 0 \leq t \leq y) \\
& =\mathbb{P}^{\eta}\left(N_{t}(x+t)=0,0 \leq t \leq y\right)=\exp \left\{-\int_{0}^{y} v([x+t, \infty)) d t\right\} .
\end{aligned}
$$

On the other hand, we know from diffusion theory [18] that

$$
\mathbb{P}^{\eta}\left(\tau_{[y, \infty)}^{W}<\tau_{(-\infty,-x]}^{W}\right)=\frac{s(0)-s(-x)}{s(y)-s(-x)}, \quad x>0,
$$

where $s$ denotes the scale function of a Brownian motion with drift $\eta[s(x)=$ $\left.\left(1-e^{-2 \eta x}\right) / 2 \eta\right]$. Comparing (3.6) and (3.7) we find for positive $x$ that $v([x, \infty))=$ $s^{\prime}(x) / s(x)$.

Now returning to the right-hand side of (3.5), we have that $L\left(\tau_{[b, \infty)}^{\Psi}\right)$ is exponentially distributed with parameter $s^{\prime}(b) / s(b)$ and hence

$$
\overline{\mathbb{E}}^{\eta}\left[e^{-(\eta-\gamma) L\left(\tau_{[b, \infty)}^{\Psi}\right)}\right]=\frac{s^{\prime}(b)}{(\eta-\gamma) s(b)+s^{\prime}(b)} .
$$

After some algebra we then recover the result in Theorem 3.2 for $\psi=1$.

Consider now the case that $\log \psi \in(0, b)$ and $\sigma=1$. Note that, $\left\{\log \Psi_{t}, t \leq\right.$ $\left.\tau_{(0, b)^{c}}^{\Psi}\right\}$ has under $\overline{\mathbb{P}}_{\psi}^{\gamma}$ the same law as $\left\{-W_{t}, t \leq \tau_{(-b, 0)^{c}}^{W}\right\}$ under $\mathbb{P}_{-\log \psi}^{\gamma}$. Set $\mathcal{A}:=$ $\mathcal{A}(\log \psi-b, \log \psi)$ equal to the event that $W$ exits the interval $\log \psi+(-b, 0)$ below and let $\mathcal{A}^{c}$ denote the complement. The strong Markov property of $\Psi$ now implies that

$$
\begin{aligned}
\overline{\mathbb{E}}_{\psi}^{\gamma}\left[e^{\left.-\lambda \tau_{[b, \infty)}^{\Psi}\right]=}\right. & \mathbb{E}^{\gamma}\left[e^{\left.-\lambda \tau_{(-\infty,-(b-\log \psi)]}^{W} \mathbf{1}_{\mathcal{A}}\right]}\right. \\
& +\mathbb{E}^{\gamma}\left[e^{-\lambda \tau_{[\log \psi, \infty)}^{W}} \mathbf{1}_{\mathcal{A}^{c}}\right] \cdot \overline{\mathbb{E}}^{\gamma}\left[e^{-\lambda \tau_{[b, \infty)}^{\Psi}}\right] .
\end{aligned}
$$

The first and second expectation follow from (3.7) applied to a Wiener process killed at an independent exponential time with parameter $\lambda$, which has as scale 
function proportional to $e^{-\gamma x} \sinh (\eta x)$. The third expectation follows from the first part of the proof. A simple algebraic exercise leads to the stated result.

In order to remove the condition $\sigma=1$, it suffices to consider the Laplace transform of the first time the process $\log \Psi^{1 / \sigma}$ enters $[b / \sigma, \infty)$.

4. Perpetual integral option. Analogously to what was done at the begining of the last section and following the procedure in [22], we combine Theorem 1.1 with the Girsanov density $s^{-1} \exp \{-r t\} S_{t}$ under $\mathbb{P}_{x}^{(r / \sigma-\sigma / 2)}$ and insist that the option holder must exercise in an almost surely finite time to achieve

$$
\Pi^{\mathrm{int}}(t, s, \varphi)=S_{t} \underset{\tau \in \mathcal{T}_{t, \infty}}{\operatorname{ess} \sup } \mathbb{E}_{x}^{(r / \sigma+\sigma / 2)}\left[e^{-\lambda \tau} \frac{\int_{t}^{\tau} S_{u} d u+\left(s \varphi+\int_{0}^{t} S_{u} d u\right)}{S_{\tau}} \mid \mathcal{F}_{t}\right] .
$$

We introduce the new stochastic process $\Phi=\left\{\Phi_{t}, t \geq 0\right\}$ with

$$
\Phi_{t}:=\frac{\int_{0}^{t} S_{u} d u+s \varphi}{S_{t}}
$$

which can easily be verified to be a Markov process. For convenience let us now assume that the Brownian motion driving the stock has been observed since some time $-M \leq 0$ and we shall interpret the constant $\varphi$ to be the quantity $s^{-1} \int_{-M}^{0} S_{u} d u$ (and assume that this is $\mathcal{F}_{0}$ measurable). Thus if $\tilde{\mathbb{P}}_{\varphi}^{\gamma}$ is the probability measure under which $W$ is a $\mathbb{P}_{0}^{\gamma}$-Brownian motion but the process $\Phi$ has value at time zero equal to $\varphi$, then it follows that

$$
\Pi^{\text {int }}(t, s, \varphi)=e^{-\lambda t} S_{t} \Pi^{\mathrm{I}}\left(\Phi_{t}\right)
$$

with

$$
\Pi^{\mathrm{I}}(\varphi)=\sup _{\tau \in \mathcal{T}_{0, \infty}} \tilde{\mathbb{E}}_{\varphi}^{(r / \sigma+\sigma / 2)}\left[e^{-\lambda \tau} \Phi_{\tau}\right]
$$

where $\tilde{\mathbb{E}}_{\varphi}^{\gamma}$ is expectation with respect to $\tilde{\mathbb{P}}_{\varphi}^{\gamma}$ and, in effect, we may now take $\Phi_{t}:=\int_{-M}^{t} S_{u} d u / S_{t}$ (which is not a function of $s$ ).

As before we have the following result, which characterizes the optimal stopping time in (4.1) as a hitting time of the process $\Phi$.

PROPOSITION 4.1. The optimal stopping time in (4.1) is a hitting time of the form

$$
\inf \left\{t \geq 0: \Phi_{t} \geq \tilde{\varphi}\right\}, \quad \tilde{\varphi} \geq 0 .
$$

Analogously as in Proposition 3.1, we can prove the form of the optimal stopping time. The finiteness of the optimal stopping time follows from the forthcoming Theorem 4.2.

The problem of pricing the perpetual integral option, just as in the case of the perpetual Russian option, is reduced to the evaluation of a Laplace transform of a stopping time of a Markov process. The following theorem essentially gives the analytical structure to the final price given in Theorem 1.5. 
THEOREM 4.2. For Borel sets B let

$$
\tau_{B}^{\Phi}=\inf \left\{t \geq 0: \Phi_{t} \in B\right\} .
$$

For $\varphi \in[0, b), \lambda \geq 0$ and $\gamma \geq 0$, we have

$$
\tilde{\mathbb{E}}_{\varphi}^{\gamma}\left[e^{-\lambda \tau_{[b, \infty)}^{\Phi}}\right]=\frac{u_{\lambda}(\varphi)}{u_{\lambda}(b)},
$$

where the function $u_{\lambda}$ is given by

$$
u_{\lambda}(x)=\int_{0}^{\infty} e^{-2 y / \sigma^{2}} y^{-\left(z_{1}+1\right)}(1+y x)^{z_{2}} d y
$$

with $z_{1}<z_{2}$ the roots of $z^{2}-(2 \gamma / \sigma) z-\left(2 \lambda / \sigma^{2}\right)=0$. In particular, $\tilde{\mathbb{P}}_{\varphi}^{\gamma}\left(\tau_{[b, \infty)}^{\Phi}<\right.$ $\infty)=1$.

We shall shortly prove this theorem but let us proceed by showing that the price of the integral option can now be quickly obtained.

Proof of TheOrem 1.5. The proof is given along the same lines as the proof of Theorem 1.4. We start by noting that Proposition 4.1, in conjunction with relation (4.1), the continuity of $\Phi$ and Theorem 4.2 with $\gamma=r / \sigma+\sigma / 2$, implies that $\Pi^{\mathrm{I}}(\varphi)$ is equal to the supremum over all $m \geq 1$ of $V_{m}^{(4)}(\varphi)$ where

$$
V_{m}^{(4)}(\varphi)=m \cdot u_{\lambda}(\varphi) / u_{\lambda}(m), \quad 0 \leq \varphi \leq m .
$$

The function $f(m):=m / u_{\lambda}(m)$ is positive and differentiable such that $f(0)=0$ and $f(m)$ decreases to 0 as $m \rightarrow \infty$. Since $u_{\lambda}$ is increasing and strictly convex it thus follows that there is a unique point in $[0, \infty)$ satisfying $f^{\prime}(m)=0$ or equivalently $u_{\lambda}(m)=m u_{\lambda}^{\prime}(m)$. The theorem is proved.

We now conclude this section by proving the main result, Theorem 4.2. We will set $\sigma=1$. The case of general $\sigma$ is reduced to the case $\sigma=1$ by noting that, by the scaling property of Brownian motion, $\left\{\Phi_{t}, t \geq 0\right\}$ has the same law under $\tilde{\mathbb{P}}_{\phi}^{\gamma}$ as $\left\{\sigma^{-2} \Phi_{\sigma^{2} t}, t \geq 0\right\}$ under $\tilde{\mathbb{P}}_{\varphi \sigma^{2}}^{\gamma / \sigma}$.

The main idea behind the proof is to take advantage of Lamperti's relation [13]; namely that for a Bessel process $R^{(\gamma)}$ with dimension $d=2(\gamma+1)$ (or index $\gamma \geq 0$ ) starting in $R^{(\gamma)}(0)=1$,

$$
\exp \left(W_{t}+\gamma t\right)=\left[R^{(2 \gamma)}\left(A_{t}^{(\gamma)} / 4\right)\right]^{2}
$$

where

$$
A_{t}^{(\gamma)}=\int_{0}^{t} \exp \left(W_{s}+\gamma s\right) d s
$$


See [18] for background on Bessel processes. Thus $\tau_{[b, \infty)}^{\Phi}$ may be considered to be of the form

$$
\tau_{[b, \infty)}^{\Phi}=\inf \left\{t \geq 0: R^{(2 \gamma)}\left(\frac{1}{4} A_{t}^{(\gamma)}\right) \leq \sqrt{\frac{4}{b}\left(\frac{1}{4} A_{t}^{(\gamma)}\right)+\frac{\varphi}{b}}\right\} .
$$

One can now see that the necessary fluctuation theory we need concerns Bessel processes. Unlike the case of the Russian option, the necessary fluctuation results we shall apply are quite deep and specific. We summarize them in the following two lemmas whose proofs can be found in [25] and [24], respectively. The first lemma is not too difficult to recover from the Girsanov theorem, but the second needs considerably more work to prove.

LEMMA 4.3. Let $\hat{P}_{x}^{\gamma}$ be the law of a Bessel process with index $\gamma$ started from $x>0$ and $\hat{E}_{x}^{\gamma}$ expectation with respect to this measure. For any stopping time $T$, define $I_{T}=\int_{0}^{T}[R(s)]^{-2} d s$ where $\{R(t): t \geq 0\}$ is a Bessel process. Suppose that $T$ is $\hat{P}_{x}^{\gamma}$-almost surely finite then, for $\lambda \geq 0$,

$$
\hat{E}_{x}^{\gamma}\left[e^{-\lambda I_{T}}\right]=\hat{E}_{x}^{\nu}\left[\left(\frac{x}{R(T)}\right)^{(\nu-\gamma)}\right]
$$

where $v=\sqrt{2 \lambda+\gamma^{2}}$.

LEMMA 4.4. Define for Bessel processes $\{R(t): t \geq 0\}$ stopping times of the form

$$
T(b)=\inf \{t \geq 0: R(t) \leq b \sqrt{1+t}\} .
$$

For any $\gamma \geq 0, x>b$ and $m \geq 0$, we have

$$
\hat{E}_{x}^{\gamma}\left[\left(\frac{1}{1+T(b)}\right)^{m}\right]=\frac{U\left(m, \gamma+1, x^{2} / 2\right)}{U\left(m, \gamma+1, b^{2} / 2\right)},
$$

where $U$ is the confluent hypergeometric Kummer's function of the second kind. That is to say that for real valued $a, b, z$,

$$
U(a, b, z)=\frac{1}{\Gamma(a)} \int_{0}^{\infty} e^{-z t} t^{a-1}(1+t)^{b-a-1} d t .
$$

(See [14] for a description of this class of functions.)

Proof of TheOREM 4.2. We give the proof for $\sigma=1$. The first thing to note is that the time change $A_{t}^{(\gamma)}$ satisfies the inverse relation (see [18])

$$
4 \int_{0}^{A_{t}^{(\gamma)} / 4}\left[R^{(2 \gamma)}(s)\right]^{-2} d s=t, \quad t \geq 0
$$


Thus we can rewrite $\tau_{[b, \infty)}^{\Phi}$ in the form

$$
\tau_{[b, \infty)}^{\Phi}=4 \int_{0}^{\tilde{T}}[R(s)]^{-2} d s
$$

under $\hat{P}_{1}^{(2 \gamma)}$, where

$$
\tilde{T}=\inf \{t: R(t) \leq \sqrt{(4 t+\varphi) / b}\} .
$$

Bessel processes have a scaling property that can be considered to be inherited from Brownian motion. Namely that if $R$ is a Bessel with index $\gamma$ with $R(0)=1$, then for any constant $c>0, R^{\prime}:=\left\{c^{-1 / 2} R(c t), t \geq 0\right\}$ is also a Bessel process with index $\gamma$ but starting from $c^{-1 / 2}$ times its original position. It thus follows after a brief calculation that $\tilde{T}$ is equal in $\hat{P}_{1}^{(2 \gamma)}$-law to $(\varphi / 4) \cdot T(\sqrt{4 / b})$ under $\hat{P}_{z}^{(2 \gamma)}$ where $z=(4 / \varphi)^{1 / 2}$. It is not hard to verify that $\lim _{a \downarrow 0} U(a, b, z)=1$, where $U$ is given in (4.6). From (4.5) we see that for $\gamma \geq 0$ and $x>b$,

$$
\hat{P}_{x}^{\gamma}(T(b)<\infty)=\lim _{m \downarrow 0} \hat{E}_{x}^{\gamma}\left[\left(\frac{1}{1+T(b)}\right)^{m} I(T(b)<\infty)\right]=1 .
$$

Hence, by the previous remark, also $\tilde{T}$ is finite $\hat{P}^{(2 \gamma)}$-almost surely and, since $R^{(\gamma)}$ with $\gamma \geq 0$ does not reach zero, we deduce that $\tilde{\mathbb{P}}_{\varphi}^{\gamma}\left(\tau_{[b, \infty)}^{\Phi}<\infty\right)=1$.

Combining this observation with Lemma 4.3, one can check that

$$
\tilde{\mathbb{E}}_{\varphi}^{\gamma}\left[e^{\left.-\lambda \tau_{[b, \infty)}^{\Phi}\right]}=\left(\frac{b}{\varphi}\right)^{-z_{1}} \hat{E}_{\sqrt{4 / \varphi}}^{\left(z_{2}-z_{1}\right)}\left[\left(\frac{1}{1+T(\sqrt{4 / b})}\right)^{-z_{1}}\right] .\right.
$$

Applying Lemma 4.4 one finds, after some algebra, the stated expression.

5. Canadization. From a financial point of view, perpetual options may be considered as rather theoretical objects, since in the real world options never have an infinite time of expiration. As we will show later, perpetual-type options can be linked to American-type options of finite expiration.

Let us consider an American-type option with finite expiration $T$ and system of payoff functions $\left\{\pi_{t}: 0 \leq t \leq T\right\}$, which are cadlag and without negative jumps. The holder of the option has the right to exercise it at any time before $T$. If the holder does not exercise before this finite time then he receives a payment $\pi_{T}$ at expiry. By considering Theorems 1.1 and 1.2 for the sequence of payments $\left\{\pi_{t \wedge T}: t \geq 0\right\}$ we have the arbitrage-free price of this American type

$$
\Pi_{T}(x)=\sup _{\tau \in \mathcal{T}_{0, T}} \mathbb{E}_{x}^{(r / \sigma-\sigma / 2)}\left[e^{-r \tau} \pi_{\tau}\right]
$$

with optimal stopping time

$$
\tau^{*}=\inf \left\{0 \leq t \leq T: \Pi_{T}(t) \leq \pi_{t}\right\},
$$


where the hedging capital, as in Section 1, is given by

$$
\Pi_{T}(t, x)=\underset{\tau \in \mathcal{T}_{t, T}}{\operatorname{ess} \sup } \mathbb{E}_{x}^{(r / \sigma-\sigma / 2)}\left[e^{-r(\tau-t)} \pi_{\tau} \mid \mathcal{F}_{t}\right]
$$

Unlike the perpetual case, the optimal stopping time is (in general) not a hitting time of a level. In many cases it will be the crossing time of a nonflat space time boundary. For this optimal exercise boundary no explicit formulas are known. For an account of the American put with finite time of expiration see, for example, [16]. Since no explicit solution is known for this problem, we consider instead a reasonable approximation. We follow the lead of [6]. The idea is to randomize $T$ in a sensible way, that is, to replace $T$ by an independent random variable.

Let $T_{1}, T_{2}, \ldots$ be a sequence of independent exponential variables with mean $T$, which are also independent of $\mathbf{F}$ and denote their probability measures and expectation, respectively, by $P$ and $E$. An $n$-step approximation is understood to mean replacing the claim process $\pi_{t \wedge T}$ by $\pi_{t \wedge T^{(n)}}$ where $T^{(n)}=n^{-1} \sum_{1}^{n} T_{i}$, which has a $\operatorname{Gamma}(n, n / T)$-distribution. Note by the strong law of large numbers $T^{(n)} \rightarrow T$ almost surely as $n$ tends to infinity. The next result shows that this approximation procedure makes sense.

PROPOSITION 5.1. Let the payments $\pi$ be $\left\{\mathcal{F}_{t}\right\}$ adapted, cadlag and without negative jumps and suppose there are $\varepsilon, C>0$ such that the family $\left\{e^{-r \tau} \pi_{\tau}: \tau \in \mathcal{T}_{0, T+\varepsilon}\right\}$ is uniformly integrable with respect to $\mathbb{P}^{(r / \sigma-\sigma / 2)}$ and $\sup _{\tau \in \mathcal{T}_{0, \infty}, u>T+\varepsilon} \mathbb{E}^{(r / \sigma-\sigma / 2)}\left[e^{-r(\tau \wedge u)} \pi_{\tau \wedge u}\right] \leq C$. Then the sequence $\left\{\Pi^{(n)}: n \geq 1\right\}$ given by

$$
\Pi^{(n)}(x)=\sup _{\tau \in \mathcal{T}_{0, \infty}} E \times \mathbb{E}_{x}^{(r / \sigma-\sigma / 2)}\left[e^{-r\left(\tau \wedge T^{(n)}\right)} \pi_{\tau \wedge T^{(n)}}\right]
$$

converges for each $x$ to $\Pi_{T}(x)$ as $n$ tends to infinity.

PROOF. For simplicity, write $g_{t}=e^{-r t} \pi_{t}, \gamma=(r / \sigma-\sigma / 2)$ and $P_{x}^{\gamma}=P \times$ $\mathbb{P}_{x}^{(r / \sigma-\sigma / 2)}$. By an extension of Theorem 1.2 to the finite expiration case, we know there exists an optimal stopping time $\tau^{*} \in \mathcal{T}_{0, T}$ such that $\Pi_{T}=\mathbb{E}^{\gamma}\left[g_{\tau^{*}}\right]$. Note that $\tau^{*} \in \mathcal{T}_{0, \infty}$ and hence $\Pi^{(n)} \geq E^{\gamma}\left[g_{\tau^{*} \wedge T^{(n)}}\right]$. By Fatou's lemma and the fact that $g$ has only nonnegative jumps, we find that

$$
\begin{aligned}
\liminf _{n \rightarrow \infty} \Pi^{(n)}(x) & \geq \liminf _{n \rightarrow \infty} E_{x}^{\gamma}\left[g_{\tau^{*} \wedge T^{(n)}}\right] \geq E_{x}^{\gamma}\left[\liminf _{n \rightarrow \infty} g_{\tau^{*} \wedge T^{(n)}}\right] \\
& \geq \mathbb{E}_{x}^{\gamma}\left[g_{\tau^{*} \wedge T}\right]=\Pi_{T}(0, x) .
\end{aligned}
$$

To finish the proof we thus have to prove that

$$
\limsup _{n \rightarrow \infty} \Pi^{(n)}(x)=\limsup _{n \rightarrow \infty} \sup _{\tau \in \mathcal{T}_{0, \infty}} E_{x}^{\gamma}\left[g_{\tau \wedge T^{(n)}}\right] \leq \Pi_{T}(x) .
$$


Using the bound on $E_{x}^{\gamma}\left[g_{\tau \wedge T^{(n)}} \mid T^{(n)}\right]$, we find that

$$
\begin{aligned}
\Pi^{(n)}(x) & \leq \sup _{\tau \in \mathcal{T}_{0, \infty}} E_{x}^{\gamma}\left[g_{\tau \wedge T^{(n)}} \mathbf{1}_{\left\{T^{(n)} \leq T+\varepsilon\right\}}\right]+\sup _{\tau \in \mathcal{T}_{0, \infty}} E_{x}^{\gamma}\left[g_{\tau \wedge T^{(n)}} \mathbf{1}_{\left\{T^{(n)}>T+\varepsilon\right\}}\right] \\
& \leq \sup _{\tau \in \mathcal{T}_{0, \infty}} \mathbb{E}_{x}^{\gamma}\left[g_{\tau \wedge(T+\varepsilon)}\right]+C \cdot P\left(T^{(n)}>T+\varepsilon\right),
\end{aligned}
$$

which after taking the lim sup for $n \rightarrow \infty$ converges to $\Pi_{T+\varepsilon}(x)$, by virtue of the fact that $T^{(n)}$ converges to $T$ almost surely. The proof is complete by showing that $\Pi_{T+\varepsilon}(x)$ tends to $\Pi_{T}(x)$ as $\varepsilon$ tends to zero. To do so, note that

$$
\begin{aligned}
\mid \sup _{\tau \in \mathcal{T}_{0, \infty}} & \mathbb{E}_{x}^{\gamma}\left[g_{\tau \wedge(T+\varepsilon)}\right]-\sup _{\tau \in \mathcal{T}_{0, \infty}} \mathbb{E}_{x}^{\gamma}\left[g_{\tau \wedge T}\right] \mid \\
\leq & \sup _{\tau \in \mathcal{T}_{0, \infty}} \mathbb{E}_{x}^{\gamma}\left[\left|g_{\tau \wedge(T+\varepsilon)}-g_{\tau \wedge T}\right|\right] \\
\quad & \sup _{\tau \in \mathcal{T}_{0, \infty}} \mathbb{E}_{x}^{\gamma}\left[\left|g_{\tau}-g_{T}\right| \mathbf{1}_{\{T<\tau \leq T+\varepsilon\}}\right] \\
\leq & \mathbb{E}_{x}^{\gamma}\left[\left|g_{\tau_{\varepsilon}}-g_{T}\right| \mathbf{1}_{\left\{T<\tau_{\varepsilon} \leq T+\varepsilon\right\}}\right]+\varepsilon,
\end{aligned}
$$

where $\tau_{\varepsilon}$ is an $\varepsilon$-optimal stopping time; that is, $\tau_{\varepsilon}$ is chosen such that

$$
\sup _{\tau \in \mathcal{T}_{0, \infty}} \mathbb{E}_{x}^{\gamma}\left[\left|g_{\tau}-g_{T}\right| \mathbf{1}_{\{T<\tau \leq T+\varepsilon\}}\right]-\varepsilon \leq \mathbb{E}_{x}^{\gamma}\left[\left|g_{\tau_{\varepsilon}}-g_{T}\right| \mathbf{1}_{\left\{T<\tau_{\varepsilon} \leq T+\varepsilon\right\}}\right] .
$$

(The existence of this $\varepsilon$-optimal stopping time follows since there is always a sequence of stopping times approximating the supremum on the left-hand side.) The expectation on the right-hand side of the previous line converges to zero by uniform integrability. Hence it follows that $\Pi_{T+\varepsilon}(x)$ can be made arbitrarily close to $\Pi_{T}(x)$ by making $\varepsilon$ sufficiently small.

REMARK. If the value function $T \mapsto \Pi_{T}$ considered as function of the expiration $T$ is a concave function, we find from Jensen's inequality that

$$
\Pi_{T}=\Pi_{E[\widetilde{T}]} \geq E\left[\Pi_{\widetilde{T}}\right] \geq \sup _{\tau \in \mathcal{T}_{0, \infty}} E \times \mathbb{E}_{x}^{(r / \sigma-\sigma / 2)}\left[e^{-r(\tau \wedge \widetilde{T})} \pi_{\tau \wedge \widetilde{T}}\right],
$$

where $\widetilde{T}$ is a random variable independent of $\mathbf{F}$ with $P$-expectation $T$.

The Canadization of an American-type option is the one-step approximation as described above. That is to say, the expiration date is randomized by an independent exponential distribution with parameter $\alpha=T^{-1}$. In all the cases we are interested in, American calls and puts, Russians and integrals, their Canadized prices are of the form

$$
\begin{aligned}
\widehat{\Pi}(\gamma) & =\sup _{\tau \in \mathcal{T}_{0, \infty}} E_{\gamma}\left[e^{-r\left(\tau \wedge T_{1}\right)} f\left(\Gamma_{\tau \wedge T_{1}}\right)\right] \\
& =\sup _{\tau \in \mathcal{T}_{0, \infty}} E_{\gamma}\left[e^{-(r+\alpha) \tau} f\left(\Gamma_{\tau}\right)+\alpha \int_{0}^{\tau} e^{-(r+\alpha) s} f\left(\Gamma_{s}\right) d s\right],
\end{aligned}
$$


where $\Gamma=\left\{\Gamma_{t}: t \geq 0\right\}$ is a continuous Markov process starting from $\gamma$ under some measure whose expectation operator is $E_{\gamma}$ and $f$ is a nonnegative, monotone increasing, convex function. It can be easily checked using Theorem 1.2 that the optimal stopping time is of the form

$$
\tau^{*}=\inf \left\{t \geq 0: \widehat{\Pi}\left(\Gamma_{t}\right) \leq f\left(\Gamma_{t}\right)\right\} .
$$

Hence on account of the properties of $f$, we can reason as in the previous sections to conclude that $\tau^{*}$ is hitting time of the Markov process $\Gamma$.

In the following examples, note that it is no longer necessary that the parameter $\lambda$ is positive in order to guarantee the existence of a solution. A finite, albeit random, expiry date removes this necessity.

5.1. One-step American put approximation $(\lambda=0)$. The first approximation $\Pi_{T_{1}}^{\text {put }}(s)$ to the price of an American put with expiration $T$

$$
\Pi_{T}^{\text {put }}(s)=\sup _{\tau \in \mathcal{T}_{0, T}} \mathbb{E}_{x}^{(r / \sigma-\sigma / 2)}\left[e^{-r \tau}\left(K-S_{\tau}\right)^{+}\right]
$$

is equal to the supremum over all $l>0$ of

$\mathbb{E}_{x}^{(r / \sigma-\sigma / 2)}\left[e^{-(r+\alpha) \tau(l)}\left(K-S_{\tau(l)}\right)^{+}\right]+\alpha \mathbb{E}_{x}^{(r / \sigma-\sigma / 2)}\left[\int_{0}^{\tau(l)} e^{-(r+\alpha) t}\left(K-S_{t}\right)^{+} d t\right]$,

where $\tau(l)=\tau_{(-\infty, l]}^{W}$ and $\alpha=T^{-1}$. Using the resolvent of the Brownian motion killed upon entering the negative halfline (see, e.g., [4])

$$
\begin{aligned}
\alpha^{-1} \mathbb{P}_{x}^{\gamma}\left(W_{e(\alpha)} \in d y, e(\alpha)<\tau_{(-\infty, 0]}^{W}\right) / d y \\
\quad=2 \Delta^{-1} e^{-x_{2} y}\left(e^{-\rho x} \sinh (\Delta x)-\mathbf{1}_{\{x \geq y\}} e^{\rho(x-y)} \sinh (\Delta x)\right),
\end{aligned}
$$

where $2 \Delta=x_{2}-x_{1}$ and $2 \rho=x_{2}+x_{1}$ with $x_{1}<x_{2}$ are the roots of $x^{2}-(1-$ $\left.2 r / \sigma^{2}\right) x-2(\alpha+r) / \sigma^{2}=0$ and $e(\alpha)$ is an independent exponential random variable with parameter $\alpha$, we find after some algebra $\Pi_{T_{1}}^{\text {put }}(s)$ to be equal to

$$
\begin{gathered}
\left(\frac{s}{K}\right)^{x_{1}} K\left(\frac{x_{2}}{x_{2}-x_{1}} \frac{\alpha}{\alpha+r}-\frac{x_{2}-1}{x_{2}-x_{1}}\right) \\
+\left(\frac{s}{l_{*}}\right)^{x_{1}} K \frac{x_{2}}{x_{2}-x_{1}} \frac{r}{r+\alpha}, \quad \text { if } s \geq K, \\
K \frac{\alpha}{r+\alpha}-s+\left(\frac{s}{K}\right)^{x_{2}} K\left(\frac{1-x_{1}}{x_{2}-x_{1}}+\frac{x_{1}}{x_{2}-x_{1}} \frac{\alpha}{\alpha+r}\right) \\
+\left(\frac{s}{l_{*}}\right)^{x_{1}} K \frac{x_{2}}{x_{2}-x_{1}} \frac{r}{r+\alpha}, \quad \text { if } s \in\left(l_{*}, K\right), \\
K-s,
\end{gathered}
$$

where the optimal exercise level is given by

$$
l_{*}=K\left(\frac{-r x_{1}}{r+\alpha-r x_{1}}\right)^{1 / x_{2}} .
$$


5.2. One-step Russian option approximation $(\lambda=0)$. According to the preceding, a first approximation to the price of a Russian option with expiry $T$

$$
\Pi_{T}^{\text {russ }}(s, \psi)=s \cdot \Pi_{T}^{\mathrm{R}}(\psi)=s \cdot \sup _{\tau \in \mathcal{T}_{0, T}} \overline{\mathbb{E}}_{\psi}^{(r / \sigma+\sigma / 2)}\left[\Psi_{\tau}\right]
$$

is equal to $\Pi_{T_{1}}^{\text {russ }}(s, \psi)=s \cdot \Pi_{T_{1}}^{\mathrm{R}}(\psi)$ where $\Pi_{T_{1}}^{\mathrm{R}}(\psi)$ is equal to the supremum over all $b>0$ of

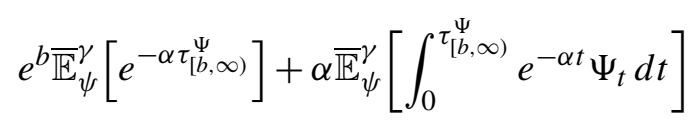

with $\alpha=T^{-1}$ and $\gamma=(r / \sigma+\sigma / 2)$. By an application of Itô's lemma to the process $\exp (-\alpha t) \Psi_{t}$, we find that

$$
\begin{aligned}
& \overline{\mathbb{E}}_{\psi}^{\gamma}\left[\int_{0}^{\tau_{[b, \infty)}^{\Psi}} e^{-\alpha t} \Psi_{t} d t\right] \\
& =-\frac{1}{r+\alpha}\left(e^{b} \overline{\mathbb{E}}_{\psi}^{\gamma}\left[e^{\left.-\alpha \tau_{[b, \infty)}^{\Psi}\right]}-\psi-\overline{\mathbb{E}}_{\psi}^{\gamma}\left[\int_{0}^{\tau_{[b, \infty)}^{\Psi}} e^{-\alpha t} S_{t}^{-1} d M_{t}\right]\right),\right.
\end{aligned}
$$

where $M_{t}=\bar{S}_{t}$. Recalling that $\bar{W}=L$ and setting $A=\left\{\tau_{\{0\}}^{\Psi}<\tau_{[b, \infty)}^{\Psi}\right\}$ the second expectation on the right-hand side can now be written

$$
\begin{aligned}
\sigma \overline{\mathbb{E}}_{\psi}^{\gamma}[ & \left.\int_{0}^{\tau_{[b, \infty)}^{\Psi}} e^{-\alpha t} \Psi_{t} d L_{t}\right] \\
= & \sigma \overline{\mathbb{E}}_{\psi}^{\gamma}\left[e^{-\alpha \tau_{\{0\}}^{\Psi}} \mathbf{1}_{A}\right] \\
& \times \int_{0}^{\infty} d t \overline{\mathbb{E}}\left[e^{-\left(\alpha+\gamma^{2} / 2\right) L^{-1}(t)+\gamma t} \mathbf{1}_{\left\{\sup _{0 \leq s<L^{-1}(t)} \bar{W}_{s}-W_{s}<b / \sigma\right\}}\right] .
\end{aligned}
$$

An application of the Girsanov theorem together with the techniques used in the proof of Theorem 3.2 concerning the two-sided exit problem yields

$$
\overline{\mathbb{E}}_{\psi}^{\gamma}\left[e^{\left.-\alpha \tau_{\{0\}}^{\psi} \mathbf{1}_{A}\right]}\right]=\psi^{\gamma / \sigma} \frac{\left(e^{b \eta / \sigma} \psi^{-\eta / \sigma}-e^{-b \eta / \sigma} \psi^{\eta / \sigma}\right)}{2 \sinh (b \eta / \sigma)},
$$

where $\eta=\sqrt{2 \alpha+\gamma^{2}}$. The integral on the right-hand side of the last but one display can also be written

$$
\begin{gathered}
\int_{0}^{\infty} d t \overline{\mathbb{P}}^{\eta}\left(\sup _{0 \leq s<L^{-1}(t)}\left(\bar{W}_{s}-W_{s}\right)<b / \sigma\right) e^{\gamma t-\eta t} \\
=\frac{\sinh (b \eta / \sigma)}{\eta \cosh (b \eta / \sigma)-\gamma \sinh (b \eta / \sigma)},
\end{gathered}
$$

where the equality follows by using the fact that the first excursion of height exceeding $b / \sigma$ appears after a length of local time which is exponentially 
distributed with parameter $s^{\prime}(b / \sigma) / s(b / \sigma)$ where the scale function $s(x)$ is taken proportional to $e^{-\eta x} \sinh (\eta x)$.

Thus, after some algebra, we find that the first approximation is given by

$$
\Pi_{T_{1}}^{\text {russ }}(s, \psi)=s \cdot\left\{\frac{r}{r+\alpha} b_{*} \frac{y_{2} \psi^{y_{1}}-y_{1} \psi^{y_{2}}}{y_{2} b_{*}^{y_{1}}-y_{1} b_{*}^{y_{2}}}+\frac{\alpha}{r+\alpha}\left(\psi+\frac{b_{*}^{y_{2}} \psi^{y_{1}}-b_{*}^{y_{1}} \psi^{y_{2}}}{y_{2} b_{*}^{y_{1}}-y_{1} b_{*}^{y_{2}}}\right)\right\},
$$

where $y_{1}=(\gamma+\eta) / \sigma$ and $y_{2}=(\gamma-\eta) / \sigma$ are the roots of $y^{2}-\left(1+2 r / \sigma^{2}\right) y-$ $2 \alpha / \sigma^{2}=0$ and the optimal exercise level $b_{*}$ is the unique solution of

$$
r\left(y_{2}\left(1-y_{1}\right) b^{y_{1}}+y_{1}\left(y_{2}-1\right) b^{y_{2}}\right)+\alpha\left(y_{2}-y_{1}\right) b^{y_{1}+y_{2}-1}=0 .
$$

Note that uniqueness follows since the function of $b$ in (5.2) is concave and differentiable with a positive derivative at 1 .

5.3. One-step integral option approximation $(\lambda=0)$. We now show how to find an approximation to the price of the integral option with expiry $T$; that is, we approximate

$$
\Pi_{T}^{\mathrm{int}}(s, \varphi)=s \cdot \Pi_{T}^{\mathrm{I}}(\varphi)=s \cdot \sup _{\tau \in \mathcal{T}_{0, T}} \tilde{\mathbb{E}}_{\varphi}^{(r / \sigma+\sigma / 2)}\left[\Phi_{\tau}\right]
$$

The first approximation $\Pi_{T_{1}}^{\mathrm{I}}$ to the price $\Pi_{T}^{\mathrm{I}}$ is given by the supremum over all $b>0$ of

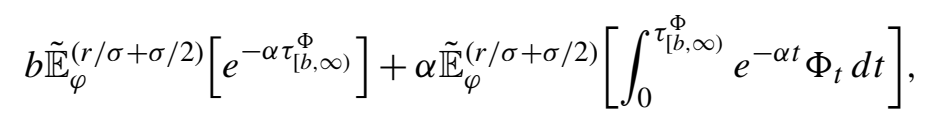

where $\alpha=T^{-1}$. An application of Itô's lemma to $\Phi_{t}$ shows that

$$
d \Phi_{t}=\left(1-r \Phi_{t}\right) d t-\sigma \Phi_{t} d W_{t}^{(r / \sigma+\sigma / 2)},
$$

where $W^{(r / \sigma+\sigma / 2)}=\left\{W_{t}-(r / \sigma+\sigma / 2) t, t \geq 0\right\}$ is a standard Wiener process under $\mathbb{P}^{(r / \sigma+\sigma / 2)}$. Applying now partial integration to $\exp (-\alpha t) \Phi_{t}$ results in

$$
\begin{aligned}
& \alpha \tilde{\mathbb{E}}_{\varphi}^{(r / \sigma+\sigma / 2)}\left[\int_{0}^{\tau_{[b, \infty)}^{\Phi}} e^{-\alpha t} \Phi_{t} d t\right] \\
& \quad=\frac{1}{\alpha+r}\left(1+\alpha \varphi-(1+\alpha b) \tilde{\mathbb{E}}_{\varphi}^{(r / \sigma+\sigma / 2)}\left[e^{\left.\left.-\alpha \tau_{[b, \infty)}^{\Phi}\right]\right)}\right.\right.
\end{aligned}
$$

Recalling formula (4.3) we find

$$
\Pi_{T_{1}}^{\mathrm{int}}(s, \varphi)=s \cdot\left\{\frac{1+\alpha \varphi}{\alpha+r}+\frac{m_{*} r-1}{\alpha+r} \frac{u_{\alpha}(\varphi)}{u_{\alpha}\left(m_{*}\right)}\right\},
$$

where, following the line of reasoning of the proof of Theorem 1.5, $m_{*}>0$ is uniquely determined by $u_{\alpha}^{\prime}\left(m_{*}\right)\left(r m_{*}-1\right)=u_{\alpha}\left(m_{*}\right) r$.

For related work on analytical approximation to Asian or integral-type options, see also [5]. 
5.4. n-step Russian option approximation $(\lambda=0)$. On a final note we consider how one would evaluate an $n$-step approximation by using a dynamic programming algorithm with the Russian option. Let $\alpha_{n}=\alpha / n$ and write $e_{i}=$ $n^{-1} T_{i}$ for $i=1, \ldots, n$. Define the subsequent stages $h_{n}, \ldots, h_{1}$ by

$$
\begin{aligned}
h_{n}(\psi) & =\sup _{\tau \in \mathcal{T}_{0, \infty}} \overline{\mathbb{E}}_{\psi}^{(r / \sigma+\sigma / 2)}\left[\Psi_{\tau \wedge e_{n}}\right] \\
& =\sup _{\tau \in \mathcal{T}_{0, \infty}} \overline{\mathbb{E}}_{\psi}^{(r / \sigma+\sigma / 2)}\left[e^{-\alpha_{n} \tau} \Psi_{\tau}+\alpha_{n} \int_{0}^{\tau} e^{-\alpha_{n} t} \Psi_{t} d t\right]
\end{aligned}
$$

and for $m=n-1, \ldots, 1$,

$$
\begin{aligned}
h_{m}(\psi) & =\sup _{\tau \in \mathcal{T}_{0, \infty}} \overline{\mathbb{E}}_{\psi}^{(r / \sigma+\sigma / 2)}\left[\Psi_{\tau \wedge \sum_{m}^{n} e_{i}}\right] \\
& =\sup _{\tau \in \mathcal{T}_{0, \infty}} \overline{\mathbb{E}}_{\psi}^{(r / \sigma+\sigma / 2)}\left[e^{-\alpha_{n} \tau} \Psi_{\tau}+\alpha_{n} \int_{0}^{\tau} e^{-\alpha_{n} t} h_{m+1}\left(\Psi_{t}\right) d t\right] .
\end{aligned}
$$

Using the Markov property it can be checked that the price $\Pi^{(n)}(x)$ of the $n$-approximation is equal to $h_{1}(\psi)$, the final outcome of the above dynamic programming algorithm, for all possible starting values $\psi$ of the Markov process. Note that each step in the dynamic programming algorithm requires a solution of a problem of the form

$$
\sup _{\tau \in \mathcal{T}_{0, \infty}} E_{\gamma}\left[e^{-\alpha_{n} \tau} f\left(\Gamma_{\tau}\right)+\alpha_{n} \int_{0}^{\tau} e^{-\alpha_{n} s} g\left(\Gamma_{s}\right) d s\right],
$$

where $g$ is another nonnegative, convex, monotone increasing function. It can be reasoned similarly to previously using Theorem 1.2 that for each stage of the algorithm, the optimal stopping time is still a hitting time. Note that the optimal stopping time for the $n$th approximation $\Pi^{(n)}$ is a randomized $\mathbf{F}$-stopping time. In future work, we will investigate the convergence properties of these randomized stopping times. The American and integral options can be dealt with similarly.

Acknowledgments. This work was inspired by discussions with F. Avram to whom we are very grateful. Both authors also thank K. Dajani and R. Doney for helpful comments and discussion.

\section{REFERENCES}

[1] Avram, F., Kyprianou, A. E. and Pistorius, M. R. (2001). Exit problems for spectrally negative Lévy processes and applications to (Canadized) Russian options. Ann. Appl. Probab. To appear.

[2] Bertoin, J. (1996). Lévy Processes. Cambridge Univ. Press.

[3] Blumenthal, R. M. (1992). Excursions of Markov Processes. Birkhäuser, Basel.

[4] Borodin, A. N. and SAlminen, P. (1996). Handbook of Brownian Motion-Facts and Formulae. Birkhäuser, Basel. 
[5] Boy Kov, Y. and Carr, P. (2001). Analytic approximation of some options. Preprint.

[6] CARR, P. (1998). Randomization and the American put. Review of Financial Studies 11 597-626.

[7] DufFie, J. D. and Harrison, J. M. (1993). Arbitrage pricing of Russian options and perpetual lookback options. Ann. Appl. Probab. 3 641-651.

[8] German, H. and Yor, M. (1993). Asian options and perpetualities. Math. Finance 3 349-375.

[9] Graversen, S. E. and PešKIR, G. (1998). On the Russian option: The expected waiting time. Theory Probab. Appl. 42 416-425.

[10] Karatzas, I. and Shreve, S. (1998). Methods of Mathematical Finance. Springer, New York.

[11] Kramkov, D. O. and Mordecki, E. (1995). Integral option. Theory Probab. Appl. 39 $162-172$.

[12] Lamberton, D. and Lapeyer, B. (1996). Introduction to Stochastic Calculus Applied to Finance. Chapman and Hall, London.

[13] Lamperti, J. W. (1972). Semistable Markov processes. Z. Wahrsch. Verw. Gebiete 22 205-225.

[14] Lebedev, N. N. (1972). Special Functions and Their Applications. Dover, New York.

[15] McKean, H. (1965). Appendix: A free boundary problem for the heat equation arising from a problem of mathematical economics. Industrial Management Review 6 32-39.

[16] Myneni, R. (1992). The pricing of the American option. Ann. Appl. Probab. 2 1-23.

[17] ØKsendal, B. (1999). Stochastic Differential Equations, 5th ed. Springer, New York.

[18] Revuz, D. and Yor, M. (1994). Continuous Martingales and Brownian Motion, 2nd ed. Springer, New York.

[19] Shepp, L. A. and Shiryaev, A. N. (1993). The Russian option: reduced regret. Ann. Appl. Probab. 3 603-631.

[20] Shepp, L. A. and Shiryaev, A. N. (1995). A new look at pricing of the "Russian option." Theory Probab. Appl. 39 103-119.

[21] ShiRyaev, A. N. (1978). Optimal Stopping Rules. Springer, Berlin.

[22] Shiryaev, A. N., Kabanov, Yu., Kramkov, D. O. and Melnikov, A. V. (1994). Toward the theory of options of both European and American types. II. Continuous time. Theory Probab. Appl. 39 61-102.

[23] Williams, D. (1979). Diffusions, Markov Processes and Martingales I: Foundations. Wiley, New York.

[24] YoR, M. (1984). On square root boundaries for Bessel processes and pole seeking Brownian motion. Stochastic Analysis and Applications. Lecture Notes in Math. 1095. Springer, New York.

[25] Yor, M. (1992). Some Aspects of Brownian Motion I. Birkhäuser, Basel.

[26] Yor, M. (1997). Some Aspects of Brownian Motion II. Birkhäuser, Basel.

DEPARTMENT OF MATHEMATICS

UNIVERSITY OF UTRECHT

BUDAPESTLAAN 6

3584 CD UTRECHT

THE NETHERLANDS

E-MAIL: kyprianou@math.uu.nl
DEPARTMENT OF MATHEMATICS

KING'S COLLEGE, LONDON

STRAND, LONDON WC2R 2LS

UNITED KINGDOM

E-MAIL: pistorius@math.uu.nl 